\title{
ON INPUT-TO-STATE STABILITY OF IMPULSIVE SYSTEMS (EXTENDED VERSION)
}

\author{
João P. Hespanha \\ Electrical and Comp. Eng. Dept. \\ Univ. California, Santa Barbara
}

\author{
Daniel Liberzon \\ Coordinated Science Lab. \\ Univ. of Illinois, Urbana-Champaign
}

\author{
Andrew R. Teel \\ Electrical and Comp. Eng. Dept. \\ Univ. California, Santa Barbara
}

\begin{abstract}
This paper introduces appropriate concepts of input-to-state stability (ISS) and integral-ISS for systems with impulsive effects. We provide a set of Lyapunov-based sufficient conditions to establish these properties. When the continuous dynamics are stabilizing but the impulsive effects are destabilizing, the impulses should not occur too frequently, which can be formalized in terms of an average dwell-time condition. Conversely, when the impulses are stabilizing and the continuous dynamics are destabilizing, there must not be overly long intervals between impulses, which is formalized in terms of a reverse average dwell-time condition. We also investigate limiting cases of systems that remain stable for arbitrarily small/large average dwell-times.
\end{abstract}

\section{INTRODUCTION}

Impulsive systems combine continuous evolution (typically described by ordinary differential equations) with impulse effects (also referred to as state jumps or resets). Stability properties of such systems have been extensively investigated in the literature; see, e.g., [3].

When investigating stability of a system, it is important to characterize the effects of external inputs. The concepts of input-to-state stability (ISS) and integral-input-to-state stability (iISS), introduced by Sontag in [17] and [18], have proved useful in this regard. Originally introduced for continuous-time systems, they were subsequently also studied for discrete-time systems [9] and switched systems [14]. The possibility of impulse effects, however, has been excluded in these works.

In this paper we study input-to-state stability properties of impulsive systems, with external signals affecting both the continuous dynamics and the state impulse map. These systems are formally defined in Section II, where we also define notions of ISS and iISS for such systems.

We provide a set of Lyapunov-based sufficient conditions to establish ISS and iISS with respect to suitable classes of impulse time sequences (see Sections III for ISS and Section VI for iISS). It is shown that when the continuous dynamics are ISS but the impulse effects are destabilizing, the impulsive system is ISS if the impulse times do not occur too frequently, which can be formalized in terms of an average dwell-time condition [7]. Conversely, when the impulses are stabilizing but the continuous dynamics are

J. Hespanha and A. Teel supported by the NSF grant CCR-0311084 and the ARO grant DAAD19-03-1-0144. D. Liberzon supported by the NSF grant ECS-0134115 and the DARPA/AFOSR MURI F49620-02-1-0325. destabilizing, the impulsive system is ISS if the impulse times satisfy a novel "reverse" average dwell-time condition, which prevents overly long intervals between impulse times.

Section IV considers impulsive systems for which both the continuous dynamics and the impulsive effects are stabilizing. Such systems are ISS regardless of how often or how seldom impulses occur. For these systems we show that there is no loss of generality in searching for Lyapunov functions that decrease at exponential rates. This result is analogous to what happens with ISS of purely discrete- or continuous-time systems (cf. [16]).

We also investigate impulsive systems that, although exhibiting destabilizing impulsive effects, remain ISS for arbitrarily small average dwell-time. Such systems typically arise when a continuous-time ISS system is perturbed by additive impulses. We also consider the dual case of impulsive systems that exhibit destabilizing continuous dynamics but remain ISS for arbitrarily large reverse average dwell-time. These systems typically can arise when a discrete-time ISS system is perturbed by a continuous drift between sampling times. Lyapunov-based stability conditions that cover both cases are provided in Section V.

The motivation to study the class of systems considered in this paper comes from multiple sources. Impulsive systems with external inputs arise naturally in control systems with communication constraints, as explicitly discussed in $[8,13$, $15,20]$. A special case of one of our results was used in [13] to analyze stability of such a system. The results presented here can be used to construct deterministic versions of the results that appeared in [20] for stochastic disturbances. Impulsive systems with inputs also describe the evolution of multiple Lyapunov functions for switched systems with inputs (even if the latter exhibit no state jumps), which in turn arise in the analysis of switching control algorithms for uncertain systems $[6,12]$.

\section{BASIC DEFINITIONS}

The general impulsive system with disturbances that we consider takes the form

$$
\left\{\begin{array}{llrl}
\dot{x}(t) & =f(x(t), w(t)), & & t \neq t_{k}, k=1,2, \ldots \\
x(t) & =g\left(x^{-}(t), w(t)\right), & & t=t_{k}, k=1,2, \ldots
\end{array}\right.
$$

where the state $x$ takes values in $\mathbb{R}^{n} ; w$ is a measurable locally bounded disturbance input taking values in $\mathbb{R}^{m}$; 
$f$ and $g$ are functions from $\mathbb{R}^{n} \times \mathbb{R}^{m} \rightarrow \mathbb{R}^{n}$, with $f$ locally Lipschitz; and $\left\{t_{1}, t_{2}, t_{3}, \ldots\right\}$ is a sequence of strictly increasing impulse times in $\left[t_{0}, \infty\right)$, where $t_{0}$ is the initial time. By construction the solution $x:\left[t_{0}, \infty\right) \rightarrow \mathbb{R}^{n}$ to (1) is right-continuous and $(\cdot)^{-}$denotes the left-limit operator, i.e., $x^{-}(t)=\lim _{s / t} x(s)$. Given a sequence $\left\{t_{k}\right\}$ and a pair of times $s, t$ satisfying $t \geq s \geq t_{0}$, we will let $N(t, s)$ denote the number of impulse times $t_{k}$ in the semi-open interval $[s, t)$.

Suppose that a sequence $\left\{t_{k}\right\}$ is given. We say that the impulsive system (1) is input-to-state stable (ISS) if there exist functions ${ }^{1} \beta \in \mathcal{K} \mathcal{L}$ and $\gamma \in \mathcal{K}_{\infty}$ such that for every initial condition and every input $w$, the corresponding solution of (1) satisfies

$$
|x(t)| \leq \beta\left(\left|x\left(t_{0}\right)\right|, t-t_{0}\right)+\gamma\left(\|w\|_{\left[t_{0}, t\right]}\right), \quad \forall t \geq t_{0}
$$

where $\|\cdot\|_{J}$ denotes the supremum norm on an interval $J$. Because of the dual role of $w$ as a continuous and discrete perturbation, it is acceptable to discard its values on a set of measure zero, provided that this set does not contain any impulse times. This can be achieved by redefining the norm of $w$ as

$$
\|w\|_{\left[t_{0}, t\right]}:=\max \left\{\operatorname{ess}_{s \in\left[t_{0}, t\right]}|w(s)|, \sup _{t_{k} \in\left[t_{0}, t\right]}\left|w\left(t_{k}\right)\right|\right\} .
$$

With this more general definition, all the subsequent developments remain valid. Considering only one function $\gamma$ in (2) leads to no loss of generality, compared to a bound in which the two terms on the right-hand-side of (3) appear weighted by different class $\mathcal{K}_{\infty}$ functions.

Since the above definition applies to a fixed sequence $\left\{t_{k}\right\}$ of impulse times, the ISS property depends on the choice of the sequence. However, it is often of interest to characterize ISS over classes of sequences $\left\{t_{k}\right\}$. To this effect, we say that the impulsive system (1) is uniformly ISS over a given class $\mathcal{S}$ of admissible sequences of impulse times if the ISS property expressed by (2) holds for every sequence in $\mathcal{S}$ with functions $\beta$ and $\gamma$ that are independent of the choice of the sequence.

The above ISS property characterizes robustness to disturbances in the $L_{\infty} / l_{\infty}$ sense. Another possibility is to consider "integral" variants, in the spirit of [18]. We say that the impulsive system (1) is integral-input-to-state stable (iISS) if there exist functions $\beta \in \mathcal{K} \mathcal{L}$ and $\alpha, \gamma \in \mathcal{K}_{\infty}$ such that for every initial condition and every input $w$, the inequality

$$
\begin{aligned}
\alpha(|x(t)|) \leq \beta\left(\left|x\left(t_{0}\right)\right|,\right. & \left.t-t_{0}\right)+\int_{t_{0}}^{t} \gamma(|w(s)|) d s \\
& +\sum_{t_{k} \in\left[t_{0}, t\right]} \gamma\left(\left|w\left(t_{k}\right)\right|\right), \quad \forall t \geq t_{0}
\end{aligned}
$$

\footnotetext{
${ }^{1}$ We say that a function $\alpha:[0, \infty) \rightarrow[0, \infty)$ is of class $\mathcal{K}$, and write $\alpha \in \mathcal{K}$, when $\alpha$ is continuous, strictly increasing, and $\alpha(0)=0$. If $\alpha$ is also unbounded, then we say it is of class $\mathcal{K}_{\infty}$ and write $\alpha \in \mathcal{K}_{\infty}$. We say that a function $\beta:[0, \infty) \times[0, \infty) \rightarrow[0, \infty)$ is of class $\mathcal{K} \mathcal{L}$, and write $\beta \in \mathcal{K} \mathcal{L}$ when $\beta(\cdot, t)$ is of class $\mathcal{K}$ for each fixed $t \geq 0$ and $\beta(s, t)$ decreases to 0 as $t \rightarrow \infty$ for each fixed $s \geq 0$.
}

holds on the domain of the corresponding solution of (1). The notion of uniform iISS over a given class $\mathcal{S}$ of impulse time sequences is defined in the same way as for ISS.

\section{SUFFICIENT CONDITIONS FOR ISS}

We say that a function $V: \mathbb{R}^{n} \rightarrow \mathbb{R}$ is a candidate exponential ISS-Lyapunov function for (1) with rate coefficients $c, d \in \mathbb{R}$ if $V$ is locally Lipschitz, positive definite radially unbounded and ${ }^{2}$

$$
\begin{aligned}
\nabla V(x) \cdot f(x, w) & \leq-c V(x)+\chi(|w|) & & \forall x, w \text { a.e. } \\
V(g(x, w)) & \leq e^{-d} V(x)+\chi(|w|) & & \forall x, w
\end{aligned}
$$

for some function $\chi \in \mathcal{K}_{\infty}$. For generality, we are assuming that $V$ is locally Lipschitz but not necessarily differentiable everywhere. However, from Rademacher's Theorem we know that the former is sufficient to guarantee that the gradient $\nabla V(x)$ of $V$ is well defined except on a set of measure zero. For this reason we qualify the quantifier in (5) with "almost everywhere." We do not require the rate coefficient $c, d$ to be non-negative and therefore $V$ will not necessarily decrease, even when $w=0$. However, when these coefficients satisfy an appropriate constraint one can use $V$ to show that the impulsive system is ISS.

Theorem 1 (uniform ISS) Let $V$ be a candidate exponential ISS-Lyapunov function for (1) with rate coefficients $c, d \in$ $\mathbb{R}$ with $d \neq 0{ }^{3}$. For arbitrary constants $\mu, \lambda>0$, let $\mathcal{S}_{\mu, \lambda}$ denote the class of impulse time sequences $\left\{t_{k}\right\}$ satisfying

$$
-d N(t, s) \leq \mu+(c-\lambda)(t-s), \quad \forall t \geq s \geq t_{0} .
$$

Then the system (1) is uniformly ISS over $\mathcal{S}_{\mu, \lambda}$.

Before proving Theorem 1, we provide some insight into the significance of condition (7).

When $d<0$, we must necessarily have $c \geq \lambda>0$ for (7) to hold. In this case, (5) says that the continuous dynamics $\dot{x}=f(x, w)$ are ISS with respect to $w$. Indeed, the existence of an ISS-Lyapunov function $V$ satisfying $\nabla V(x) \cdot f(x, w) \leq-\alpha(V(x))+\chi(|w|)$ with $\alpha, \chi \in \mathcal{K}_{\infty}$ is equivalent to ISS [19], and taking $\alpha$ to be linear is no loss of generality [16].

Since $d<0$, the impulses can potentially destroy ISS, and we must require that they not happen too frequently. Not surprisingly, in this case the condition (7) enforces an upper bound on the number of impulses times: for $c=\lambda$ it only holds when the number of impulse times is no larger than $N_{0}:=\frac{\mu}{|d|}$ and for $c>\lambda$ it can be re-written as

$$
N(t, s) \leq \frac{t-s}{\tau^{*}}+N_{0}, \quad \forall t \geq s \geq t_{0}
$$

\footnotetext{
${ }^{2}$ Taking the same function $\chi$ in (5) and (6) is no loss of generality, because we can always consider the maximum of two functions; however, it is also easy to treat the case of two different functions, which would lead to slightly more complicated notation but less conservative estimates.

${ }^{3}$ When $d=0$, Theorem 1 can still be applied because (6) also holds for every $d<0$. This case is closely related to the results in Section V.
} 
where $\tau^{*}:=\frac{|d|}{c-\lambda}, N_{0}:=\frac{\mu}{\mid d}$. This corresponds to the concept of average dwell-time for switched systems introduced in [7]. The special case $N_{0}=1$ reduces to a dwell-time condition in which consecutive impulses must be separated by at least $\tau^{*}$ units of time.

When $d>0$, the condition (7) only poses a constraint when $\lambda>c$. In this case, it enforces a lower bound on the number of impulse times and it can be re-written as

$$
N(t, s) \geq \frac{t-s}{\tau^{*}}-N_{0}, \quad \forall t \geq s \geq t_{0},
$$

where $\tau^{*}:=\frac{d}{\lambda-c}, N_{0}:=\frac{\mu}{d}$. It now corresponds to a reverse average dwell-time condition that demands, on average, at least one impulse per interval of length $\tau^{*}>0$. With $d>0,(6)$ says that the discrete dynamics $x(k+1)=$ $g(x(k), w(k))$ are ISS with respect to $w$. When we have $c<0$, the continuous flow can potentially destroy ISS, so we must require flows to be persistently interrupted by impulses through the reverse average dwell-time condition. We recall that, according to [1, Theorem 1], the condition (5) with $c<0$ is equivalent to forward completeness (bounded response to bounded inputs) of the continuous dynamics. The following result follows from the discussion above:

Corollary 2 (average dwell-time ISS) Let $V$ be a candidate exponential ISS-Lyapunov function for (1) with rate coefficients $c, d \in \mathbb{R}$.

1) Let $\mathcal{S}_{\mathrm{avg}}\left[\tau^{*}, N_{0}\right]$ denote the class of average dwell-time impulse time sequences that satisfy (8). When $d<0$, (1) is uniformly ISS over $\mathcal{S}_{\text {avg }}\left[\tau^{*}, N_{0}\right]$, for every $\tau^{*}>$ $|d| / c$ and $N_{0}>0$.

2) Let $\mathcal{S}_{\mathrm{r}-\mathrm{avg}}\left[\tau^{*}, N_{0}\right]$ denote the class of reverse average dwell-time impulse time sequences that satisfy (9). When $d>0$, (1) is uniformly ISS over $\mathcal{S}_{\mathrm{r}-\mathrm{avg}}\left[\tau^{*}, N_{0}\right]$, for every $\tau^{*}<d /(-c)$ and $N_{0}>0$.

Proof of TheOrem 1. Dividing both sides of (7) by $1+\varepsilon>$ 0 , we conclude that

$$
-\bar{d} N(t, s)-\bar{c}(t-s) \leq \bar{\mu}-\bar{\lambda}(t-s), \quad \forall t \geq s \geq t_{0} .
$$

where

$$
\bar{d}:=\frac{d}{1+\varepsilon}, \quad \bar{c}:=\frac{c-\frac{\lambda}{2}}{1+\varepsilon}, \quad \bar{\mu}:=\frac{\mu}{1+\varepsilon}, \quad \bar{\lambda}:=\frac{\lambda}{2(1+\varepsilon)} .
$$

Moreover, we can always choose $\varepsilon \in \mathbb{R}$ sufficiently small so that $\bar{c}<c$ and $\bar{d}<d$. We can then rewrite (5) as

$$
\nabla V(x) \cdot f(x, w) \leq-\bar{c} V(x)-(c-\bar{c}) V(x)+\chi(|w|),
$$

and conclude that

$$
(c-\bar{c}) V(x) \geq \chi(|w|) \Rightarrow \nabla V(x) \cdot f(x, w) \leq-\bar{c} V(x),
$$

$\forall x, w$ a.e.. Similarly, from (6) we also conclude that

$$
\left(e^{-\bar{d}}-e^{-d}\right) V(x) \geq \chi(|w|) \Rightarrow V(g(x, w)) \leq e^{-\bar{d}} V(x),
$$

$\forall x, w$ a.e. Let

$$
\check{t}_{1}:=\min \left\{t \geq t_{0}: V(x(t)) \leq a \chi\left(\|w\|_{\left[t_{0}, t\right]}\right)\right\} \leq \infty,
$$

$a:=\frac{1}{\min \left\{c-\bar{c}, e^{-d}-e^{-d}\right\}}>0$. This is well defined because both $x(t)$ and $\|w\|_{\left[t_{0}, t\right]}$ are right-continuous in $t$ (recall the definition (3) of the norm). In view of (11) and (12),

$$
\dot{V}(x(t)) \leq-\bar{c} V(t), \quad \forall t \in\left[t_{0}, \check{t}_{1}\right) \text { a.e. }
$$

along the continuous dynamics ${ }^{4}$ and $V(x) \leq e^{-\bar{d}} V\left(x^{-}\right)$ during the impulses. Thus on this time interval we have the bound

$$
V(x(t)) \leq e^{-\bar{d} N\left(t, t_{0}\right)-\bar{c}\left(t-t_{0}\right)} V\left(x\left(t_{0}\right)\right), \quad \forall t \in\left[t_{0}, \check{t}_{1}\right)
$$

(cf. [11, Theorem 1.10.2]). Combining this with (10), we conclude that

$$
V(x(t)) \leq e^{k-\bar{\lambda}\left(t-t_{0}\right)} V\left(x\left(t_{0}\right)\right), \quad \forall t \in\left[t_{0}, \check{t}_{1}\right) .
$$

When $\check{t}_{1}=+\infty$, an ISS bound could already be deduced from here using standard arguments. Otherwise, let $\hat{t}_{1}:=$ $\inf \left\{t>\check{t}_{1}: V(x(t))>a \chi\left(\|w\|_{\left[t_{0}, t\right]}\right)\right\} \leq \infty$. By construction, we have

$$
V(x(t)) \leq a \chi\left(\|w\|_{\left[t_{0}, t\right]}\right), \quad \forall t \in\left[\check{t}_{1}, \hat{t}_{1}\right) .
$$

Next, let $\check{t}_{2}:=\min \left\{t>\hat{t}_{1}: V(x(t)) \leq a \chi\left(\|w\|_{\left[t_{0}, t\right]}\right)\right\} \leq$ $\infty$. Repeating the argument used to establish (14), with $\hat{t}_{1}$ in place of $t_{0}$, we obtain

$$
\begin{aligned}
V(x(t)) & \leq e^{\bar{\mu}-\bar{\lambda}\left(t-\hat{t}_{1}\right)} V\left(x\left(\hat{t}_{1}\right)\right) \\
& \leq e^{\bar{\mu}-\bar{\lambda}\left(t-\hat{t}_{1}\right)}\left(e^{-d} V\left(x^{-}\left(\hat{t}_{1}\right)\right)+\chi\left(\left|w\left(\hat{t}_{1}\right)\right|\right)\right) \\
& \leq e^{\bar{\mu}-\bar{\lambda}\left(t-\hat{t}_{1}\right)}\left(a e^{-d} \chi\left(\|w\|_{\left[t_{0}, t\right]}\right)+\chi\left(\left|w\left(\hat{t}_{1}\right)\right|\right)\right) \\
& \leq e^{\bar{\mu}}\left(a e^{-\bar{d}}+1\right) \chi\left(\|w\|_{\left[t_{0}, t\right]}\right) \quad \forall t \in\left[\hat{t}_{1}, \check{t}_{2}\right) .
\end{aligned}
$$

Arguing in the same way for all future times, we arrive at the bound

$$
\begin{aligned}
& V(x(t)) \leq e^{\bar{\mu}-\bar{\lambda}\left(t-t_{0}\right)} V\left(x\left(t_{0}\right)\right)+ \\
& \qquad e^{\bar{\mu}}\left(a e^{-\bar{d}}+1\right) \chi\left(\|w\|_{\left[t_{0}, t\right]}\right), \quad \forall t \geq t_{0} .
\end{aligned}
$$

The ISS estimate (2) follows from this by standard arguments. Namely, since $V$ is positive definite and radially unbounded, it satisfies $\alpha_{1}(|x|) \leq V(x) \leq \alpha_{2}(|x|)$ for some $\alpha_{1}, \alpha_{2} \in \mathcal{K}_{\infty}$, which allows us to convert a $\mathcal{K} \mathcal{L}$ estimate for $V(x)$ into that for $|x|$. Uniformity is also clear, since the final bound on $V$ and hence the functions $\beta$ and $\gamma$ in (2) do not depend on the particular choice of the impulse time sequence.

Remark 1 We can see from the proof of Theorem 1 that the condition (7) on the impulse rate only needs to hold for times $t$ on which

$$
V(x(t))>\frac{\chi\left(\|w\|_{\left[t_{0}, t\right]}\right)}{\min \left\{c-\bar{c}, e^{-\bar{d}}-e^{-d}\right\}} .
$$

\footnotetext{
${ }^{4}$ The function $t \mapsto V(x(t))$ is absolutely continuous because $V$ is locally Lipschitz and $x$ absolutely continuous on $\left[t_{0}, \breve{t}_{1}\right)$. Therefore $V(x(t))$ has time-derivative almost everywhere in this interval.
} 


\section{NON-EXPONENTIAL ISS-LYAPUNOV FUNCTIONS}

When the rate coefficients of a candidate exponential ISS-Lyapunov function are both positive, we have ISS for arbitrary impulse time sequences, because (7) poses no constraints on the impulse time sequences, as long as we choose $\lambda \leq c$. In this case, we may ask whether there would be an advantage in allowing a non-linear dependence on $V$ in the right-hand-sides of (5)-(6). In particular, we can ask if it would be possible to show that a larger class of systems is ISS for arbitrary impulse time sequences by simply demanding the existence of a "non-exponential" locally Lipschitz, positive definite, radially unbounded Lyapunov function $U$ that satisfies ${ }^{5}$

$$
\begin{aligned}
\nabla U(x) \cdot f(x, w) & \leq-\alpha(U(x))+\chi(|w|) \quad \forall x, w \text { a.e. } \\
U(g(x, w)) & \leq(i d-\alpha)(U(x))+\chi(|w|) \quad \forall x, w
\end{aligned}
$$

with $\alpha, \chi \in \mathcal{K}_{\infty}$. The following result answers this question in the negative:

Theorem 3 (exponential vs. non-exponential) The following two statements are equivalent:

1) There exists a locally Lipschitz, positive definite, radially unbounded function $U: \mathbb{R}^{n} \rightarrow \mathbb{R}$ that satisfies (15)-(16).

2) There exists a candidate exponential ISS-Lyapunov function $V$ for (1) with positive rate coefficients.

In either case, (1) is uniformly ISS over all impulse time sequences.

Proof of Theorem 3. The implication from 2 to 1 is trivial. To prove the converse we explicitly construct a candidate exponential ISS-Lyapunov function $V$ from a nonexponential one $U$. Let $V(x):=\kappa(U(x)), \forall x$, where $\kappa \in$ $\mathcal{K}_{\infty}$ is chosen to be continuously differentiable with $\kappa^{\prime}$ is nonnegative and nondecreasing, and

$$
\kappa^{\prime}(s) \alpha(s) \geq 2 \kappa(s), \quad \forall s \geq 0 .
$$

Such a function is constructed in [16, pp. 22-23]. From (17) and (15), we conclude that

$$
\begin{aligned}
& \nabla V(x) \cdot f(x, w)=\kappa^{\prime}(U(x)) \nabla U(x) \cdot f(x, w) \\
& \leq-\kappa^{\prime}(U(x)) \alpha(U(x))+\kappa^{\prime}(U(x)) \chi(|w|) \\
& \leq-V(x)-\frac{\kappa^{\prime}(U(x)) \alpha(U(x))}{2}+\kappa^{\prime}(U(x)) \chi(|w|),
\end{aligned}
$$

$\forall x, w$ a.e. When $\alpha(U(x)) \leq 2 \chi(|w|)$, we have

$$
\kappa^{\prime}(U(x)) \chi(|w|) \leq \kappa^{\prime} \circ \alpha^{-1}(2 \chi(|w|)) \chi(|w|)=: \bar{\chi}(|w|)
$$

and when $\alpha(U(x))>2 \chi(|w|)$,

$$
-\frac{\kappa^{\prime}(U(x)) \alpha(U(x))}{2}+\kappa^{\prime}(U(x)) \chi(|w|) \leq 0 .
$$

\footnotetext{
${ }^{5}$ Taking the same functions $\alpha$ and $\chi$ in (15) and (16) is no loss of generality, because we could always consider the minimum of the two $\alpha^{\prime}$ a and the maximum of the two $\chi$ 's.
}

In either case, we conclude from (18) that

$$
\nabla V(x) \cdot f(x, w) \leq-V(x)+\bar{\chi}(|w|), \quad \forall x, w \text { a.e. }
$$

On the other hand, using (17), the Mean Value Theorem and the fact that $\kappa^{\prime}$ is nondecreasing, we conclude that

$$
\begin{aligned}
2 \kappa(s) \leq \frac{\kappa^{\prime}(s) \alpha(s)}{2}+\kappa(s) & \leq \kappa\left(s+\frac{\alpha(s)}{2}\right) \\
= & \kappa \circ\left(i d+\frac{\alpha}{2}\right)(s), \quad \forall s \geq 0 .
\end{aligned}
$$

Specializing this inequality for ${ }^{6} s:=\left(i d-\frac{\alpha}{2}\right)(r), r \geq 0$ and using the fact that

$$
\left(i d-\frac{\alpha}{2}\right)(r) \leq\left(i d+\frac{\alpha}{2}\right)^{-1}(r), \quad \forall r \geq 0,
$$

we further obtain

$$
2 \kappa \circ\left(i d-\frac{\alpha}{2}\right)(r) \leq \kappa(r), \quad \forall r \geq 0 .
$$

From the definition of $V$ and (16), we conclude that

$$
V(g(x, w)) \leq \kappa((i d-\alpha)(U(x))+\chi(|w|) \mid)
$$

$\forall x, w$. When $\alpha(U(x)) \leq 2 \chi(|w|)$, we have

$$
\begin{array}{r}
\kappa((i d-\alpha)(U(x))+\chi(|w|) \mid) \leq \kappa(U(x)+\chi(|w|) \mid) \\
\leq \kappa\left(\alpha^{-1}(2 \chi(|w|))+\chi(|w|) \mid\right)=: \tilde{\chi}(|w|)
\end{array}
$$

and when $\alpha(U(x))>2 \chi(|w|)$,

$$
\begin{aligned}
\kappa((i d-\alpha)(U(x))+ & \chi(|w|) \mid) \\
& \leq \kappa \circ\left(i d-\frac{\alpha}{2}\right)(U(x)) \leq \frac{V(x)}{2},
\end{aligned}
$$

by virtue of (20). In either case, we conclude from (21) that

$$
V(g(x, w)) \leq \frac{V(x)}{2}+\tilde{\chi}(|w|), \quad \forall x, w .
$$

This finishes the proof since (19) and (22) show that $V$ is a candidate exponential ISS-Lyapunov function $V$ with positive rate coefficients.

It should be clear from the proof of Theorem 3 that if (16) is replaced by $U(g(x, w)) \leq U(x), \forall x, w$, i.e., if the impulses are "neutral" rather than "helpful" for ISS, then there exists a candidate exponential ISS-Lyapunov function $V$ with rate coefficients $c=1, d=0$, for which the $\chi$ term is absent from (6). In this case, it is straightforward to prove that (1) is also uniformly ISS over all impulse time sequences.

The dual case-when the continuous dynamics are "neutral" and the impulses are "helpful" for ISS-is also of interest, but then we must require that the impulses are persistent in the sense of (9) with $\tau^{*}>0$ arbitrary. Taking into account Remark 1, here is one way to state the result.

\footnotetext{
${ }^{6}$ The function $i d-\frac{\alpha}{2}$ must be nonnegative, otherwise we would obtain a contradiction between (16) and the positive definiteness of $U$.
} 
Theorem 4 (neutral continuous dynamics) Suppose that there exist a locally Lipschitz, positive definite, radially unbounded function $V: \mathbb{R}^{n} \rightarrow \mathbb{R}$ such that

$$
\nabla V(x) \cdot f(x, w) \leq 0, \quad \forall x, w \text { a.e. }
$$

and class $\mathcal{K}_{\infty}$ functions $\alpha$ and $\rho$ such that

$$
V(x) \geq \rho(|w|) \Rightarrow V(g(x, w))-V(x) \leq-\alpha(V(x))
$$

and

$$
V(x) \leq \rho(r) \text { and }|w| \leq r \Rightarrow V(g(x, w)) \leq \rho(r) .
$$

Fix an arbitrary positive integer $N_{0}$ and an arbitrary positive real number $\tau^{*}$. Let $\mathcal{S}_{N_{0}, \tau^{*}}$ denote the class of impulse time sequences $\left\{t_{k}\right\}$ with the following property: for each $t>t_{0}$ such that $V(x(\bar{t})) \geq \rho\left(\|w\|_{\left[t_{0}, \bar{t}\right]}\right)$ for all $\bar{t} \in\left[t_{0}, t\right]$, the number $N\left(t, t_{0}\right)$ of impulse times in the interval $\left[t_{0}, t\right]$ satisfies the inequality (9) with $s=t_{0}$. Then the system (1) is uniformly ISS over $\mathcal{S}_{N_{0}, \tau^{*}}$.

\section{Proof OF THEOREM 4. Let}

$$
\check{t}:=\min \left\{t \geq t_{0}: V(x(t)) \leq \rho\left(\|w\|_{\left[t_{0}, t\right]}\right)\right\} \leq \infty
$$

(this is well defined in view of right-continuity). By virtue of (23), we have $V(x)-V\left(x^{-}\right) \leq-\alpha\left(V\left(x^{-}\right)\right)$at each impulse time in the interval $\left[t_{0}, \breve{t}\right)$. Therefore, there exists a function $\bar{\beta} \in \mathcal{K} \mathcal{L}$ such that

$$
V(x(t)) \leq \bar{\beta}\left(V\left(x\left(t_{0}\right)\right), N\left(t, t_{0}\right)\right), \quad \forall t \in\left[t_{0}, \check{t}\right)
$$

(cf. [9]). Invoking (9), we have

$$
\begin{aligned}
& V(x(t)) \leq \bar{\beta}\left(V\left(x\left(t_{0}\right)\right), \max \left\{0, \frac{t-t_{0}}{\tau^{*}}-N_{0}\right\}\right) \\
& =: \beta\left(V\left(x\left(t_{0}\right)\right), t-t_{0}\right), \quad \forall t \in\left[t_{0}, \check{t}\right) .
\end{aligned}
$$

Next, (24) applied with $r:=\|w\|_{\left[t_{0}, t\right]}$ at each impulse time guarantees that $V(x(t)) \leq \rho\left(\|w\|_{\left[t_{0}, t\right]}\right), \forall t \geq \check{t}$. Combining the two inequalities gives the ISS estimate.

The conditions (23) and (24) are both satisfied if we have

$$
V(g(x, w))-V(x) \leq-\bar{\alpha}(V(x))+\chi(|w|)
$$

for some $\bar{\alpha}, \chi \in \mathcal{K}_{\infty}$. Indeed, letting $\rho(r):=\bar{\alpha}^{-1}(2 \chi(r))$, we see that (23) holds with $\alpha:=\bar{\alpha} / 2$. Decreasing $\bar{\alpha}$ if necessary, assume with no loss of generality that $i d-\bar{\alpha} \in \mathcal{K}$. We then have

$$
\begin{aligned}
V(x) & \leq \bar{\alpha}^{-1}(2 \chi(r)),|w| \leq r \quad \Rightarrow \\
V(g(x, w)) & \leq(i d-\bar{\alpha})\left(\bar{\alpha}^{-1}(2 \chi(r))\right)+\chi(|w|) \\
& <\bar{\alpha}^{-1}(2 \chi(|w|))
\end{aligned}
$$

and so (24) holds with the same $\rho$. Moreover, (23) implies (25) - and consequently (24) - if the impulse map $g$ is continuous. Still, it is useful to write two separate conditions (23) and (24) if we want a less conservative result. The former condition coupled with (9) is the main ingredient for obtaining ISS, while the latter is automatically enforced if, for example, impulses can only decrease $V(x)$.

\section{ISS FOR ARBITRARY AVERAGE DWELL-TIMES}

This section addresses two classes of systems for which one may not have uniform ISS over all impulse sequences, but one still has ISS over classes of impulse sequences with arbitrarily small average dwell-time or arbitrarily large reverse average dwell-time.

Impulsive systems often arise out of applying impulsive perturbations to an ISS continuous-time system. This occurs, e.g., in [13, Section 4] in the context of control with limited information. For such systems, one should not expect uniform ISS over all impulse sequences, but one can still ask "how often" can these perturbations occur without destroying ISS. The first part of Corollary 2 provides an answer to this question in terms of a minimum average dwell-time $\tau^{*}>$ $|d| / c$. However, it prompts the question of whether or not we could have uniform ISS for an arbitrary small (but nonzero) average dwell-time $\tau^{*}>0$. Motivated by this observation, we say that the impulsive system (1) is ISS for arbitrarily small average dwell-time (a.d.t) when it is uniformly ISS over every class $\mathcal{S}_{\text {avg }}\left[\tau^{*}, N_{0}\right]$ of average dwell-time impulse time sequences that satisfy (8) with $\tau^{*}>0, N_{0}<\infty$.

Alternatively, impulsive systems can arise out of applying continuous-time perturbations to an ISS discrete-time system. This occurs, e.g., in [20] in the context of networked control systems ${ }^{7}$. In this case, one may ask for "how long" can the system flow between jumps without destroying ISS. The second part of Corollary 2 provides an answer to this question in terms of a maximum reverse average dwell-time $\tau^{*}:=d /(-c), \lambda>0$. However, also in this case one may have uniform ISS for an arbitrary large (but finite) reverse average dwell-time $\tau^{*}>0$. Motivated by this observation, we say that the impulsive system (1) is ISS for arbitrarily large reverse a.d.t. when it is uniformly ISS over every class $\mathcal{S}_{\text {r-avg }}\left[\tau^{*}, N_{0}\right]$ of reverse average dwell-time impulse time sequences that satisfy (9) with $\tau^{*}, N_{0}<\infty$.

To address these questions it is convenient to introduce the following terminology: We say that $V: \mathbb{R}^{n} \rightarrow \mathbb{R}$ is non-expansive for the impulse map $g(x, w)$ when, for every $d<0$, there exists a function $\chi \in \mathcal{K}_{\infty}$ such that

$$
V(g(x, w)) \leq e^{-d} V(x)+\chi(|w|) \quad \forall x, w .
$$

This terminology is motivated by the observation that these functions must necessarily satisfy $V(g(x, 0)) \leq V(x), \forall x$. Alternatively, we say that a locally Lipschitz function $V$ is non-expansive for the vector field $f(x, w)$ when, for every $c<0$, there exists a function $\chi \in \mathcal{K}_{\infty}$ such that

$$
\nabla V(x) \cdot f(x, w) \leq-c V(x)+\chi(|w|) \quad \forall x, w \text { a.e. }
$$

These functions must always satisfy $\nabla V(x) \cdot f(x, 0) \leq 0$, $\forall x$ a.e. The following result follows from the formulas given above for $\tau^{*}$ :

\footnotetext{
${ }^{7}$ The analysis in [20] deals with stochastic disturbances $w$ and considers more general vector fields. A deterministic version of the framework in [20] with marginally stable processes leads to the class of systems considered here.
} 
Corollary 5 (ISS for arbitrary a.d.t) Let $V$ be a candidate exponential ISS-Lyapunov function for (1).

1) If $V$ is non-expansive for the impulse map $g(x, w)$, then (1) is ISS for arbitrarily small a.d.t.

2) If $V$ is non-expansive for the vector field $(f, w)$, then (1) is ISS for arbitrarily large reverse a.d.t.

The remaining of this section is devoted to the question of whether or not a given function $V$ is non-expansive.

\section{A. Non-expansiveness for impulse maps}

To state the following result we say that a function $h(x, w)$ has linear growth on $w$ uniformly over $x$ if

$$
\exists L>0 \quad: \quad|h(x, w)| \leq L|w|, \quad \forall x, w .
$$

Theorem 6 (non-expansive for impulse maps) Assume that $g(x, w)-x$ has linear growth on $w$ uniformly over $x$. A locally Lipschitz, positive definite, radially unbounded function $V: \mathbb{R}^{n} \rightarrow \mathbb{R}$ is non-expansive for the impulse map $g(x, w)$ if any of the following conditions holds:

C1 $\forall d<0$, there exists a function $\bar{\chi} \in \mathcal{K}_{\infty}$ such that

$$
V(x+y) \leq e^{-d} V(x)+\bar{\chi}(|y|), \quad \forall x, y .
$$

C2 there exists a function $\alpha \in \mathcal{K}_{\infty}$ such that ${ }^{8}$

$$
\alpha(V(x))|\nabla V(x)| \leq V(x), \quad \forall x \text { a.e. }
$$

C3 $\frac{V(x)}{|\nabla V(x)|}$ is radially unbounded a.e.

C4 $V$ is quadratic or, more generally, takes the form

$$
V(x)=\sum_{i=1}^{m}\left(x^{T} P_{i} x\right)^{i}, \quad P_{i} \geq 0, i \in\{1, \ldots, m\}
$$

where $m$ is a positive integer.

Remark 2 Theorem 6 indicates that there is a broad class of positive definite functions $V$ that are non-expansive for impulse maps for which $g(x, w)-x$ has linear growth. Indeed, C3 simply requires that the gradient of $V$ be dominated by $V$ itself, which happens for large classes of polynomial functions as well as for every homogeneous function. One may then wonder if there are interesting systems with such impulse maps that are ISS for a given average dwell-time $\tau^{*}>0$ but are not ISS for arbitrarily small a.d.t. The answer is affirmative and a simple example is given by

$$
\begin{cases}\dot{x}=-\operatorname{sat}(x), & t \neq t_{k}, k=1,2, \ldots \\ x=x^{-}+\operatorname{sat}(w), & t=t_{k}, k=1,2, \ldots,\end{cases}
$$

where sat $(\cdot)$ denotes the saturation function limited at \pm 1 and with unit slope on $[-1,1]$. This system is uniformly ISS

\footnotetext{
${ }^{8}$ Since $V$ is positive definite, one could replace $\alpha(V(x))$ by $\alpha(\|x\|)$ in
}

over the class of average dwell-time impulse time sequences that satisfy (8) for any $\tau^{*}>1$ because

$$
V(x):= \begin{cases}x^{2} & |x| \leq 1 \\ e^{2(|x|-1)} & |x|>1\end{cases}
$$

is a candidate exponential ISS-Lyapunov function with rate coefficients $c=2$ and $d=-2$. However, it is not ISS for arbitrarily small a.d.t. since $x$ can explode with bounded disturbances, provided that the impulse times are closely spaced. As expected, the function (30) does not satisfy (29).

\section{Corollary 7 (GES vs. ISS for arbitrarily small a.d.t.)}

Impulsive systems of the following form are always ISS for arbitrarily small a.d.t.:

$$
\begin{cases}\dot{x}=f_{1}(x)+f_{2}(w), & t \neq t_{k}, k=1,2, \ldots \\ x=g\left(x^{-}, w\right), & t=t_{k}, k=1,2, \ldots\end{cases}
$$

where $\dot{x}=f_{1}(x)$ is globally exponentially stable, $f_{1}$ is globally Lipschitz, $f_{2}(0)=0$, and $g(x, w)-x$ has linear growth on $w$ uniformly over $x$. This includes the case of linear continuous dynamics.

ProOF OF COROLlary 7 [10, Theorem 4.14] guarantees the existence of a locally Lipschitz function $V$ satisfying $a_{1}|x|^{2} \leq V(x) \leq a_{2}|x|^{2}, \nabla V(x) \cdot f_{1}(x) \leq-a_{3}|x|^{2}$, and $|\nabla V(x)| \leq a_{4}|x|$ a.e., where $a_{i}>0, i=1,2,3,4$. This implies that

$$
\nabla V(x) \cdot\left(f_{1}(x)+f_{2}(w)\right) \leq-a_{3}|x|^{2}+a_{4}|x| f_{2}(w),
$$

from which (5) follows by square completion. On the other hand, $V$ is non-expansive for the impulse map $g(x, w)$ due to condition C3 and the result follows from Corollary 5.

Proof of Theorem 6. C1. Consider an arbitrary $d<0$. Using (28) and the fact that $g(x, w)-x$ has linear growth, we can write

$$
\begin{gathered}
V(g(x, w))-e^{-d} V(x)=V(x+g(x, w)-x)-e^{-d} V(x) \\
\leq \bar{\chi}(|g(x, w)-x|) \leq \bar{\chi}(L|w|)=: \chi(|w|),
\end{gathered}
$$

from which (26) follows.

C2. Without loss of generality we assume that $\alpha$ is locally Lipschitz, as if this were not the case we could replace $\alpha$ by a smaller locally Lipschitz function in $\mathcal{K}_{\infty}$. We show that $\mathrm{C} 2$ implies $\mathrm{C} 1$. To this effect, for an arbitrary $d<0$, let $\bar{\chi} \in \mathcal{K}_{\infty}$ be such that

$$
|x| \geq \bar{\chi}(|y|) \quad \Rightarrow \quad \alpha(V(x)) \geq-|y| / d .
$$

Such a function exists because, since $V$ is positive definite and radially unbounded, there exists a function $\alpha_{1} \in \mathcal{K}_{\infty}$ such that $\alpha_{1}(|x|) \leq V(x)$. It is then straightforward to verify that one possible $\bar{\chi}$ is given by

$$
\bar{\chi}(s):=\alpha_{1}^{-1}\left(\alpha^{-1}(-s / d)\right), \quad \forall s \geq 0 .
$$

We start by taking some $x, y \in \mathbb{R}^{n} \backslash\{0\}$ such that

$$
|x| \geq \bar{\chi}(|y|), \quad V(x+y)>V(x) .
$$


Without loss of generality we assume that $\bar{\chi}(|y|) \geq 2|y|$ and therefore $|x| \geq 2|y|$. This guarantees that $x+y \neq 0$ because $g(x, w)-x$ has linear growth. Since $V$ is positive definite, it must be true that

$$
\mu:=\inf _{s \in[0,1]} V(x+s y)>0 .
$$

Defining $v(s):=V(x+s y), \forall s \in[0,1]$, we conclude that $v(0)=V(x)>0$ and

$$
v^{\prime}(s) \leq \nabla V(x+s y) \cdot y, \quad \forall s \in[0,1] \text { a.e. }
$$

We then conclude from (29) that

$$
v(0)=V(x)>0, \quad v^{\prime}(s) \leq \frac{v(s)|y|}{\alpha(v(s))}, \quad \forall s \in[0,1] \text { a.e. }
$$

Suppose now that we define $u:[0,1] \rightarrow[\mu, \infty)$ as the solution to

$$
u(0)=V(x)>0, \quad u^{\prime}(s)=\frac{u(s)|y|}{\alpha(u(s))}, \quad \forall s \in[0,1] .
$$

Since $\frac{u}{\alpha(u)}|y|$ is locally Lipschitz on $[\mu, \infty)$ we conclude from [11, Theorem 1.10.2] that

$$
v(s) \leq u(s), \quad \forall s \in\left[0, s^{*}\right),
$$

where $\left[0, s^{*}\right]$ denotes any interval for which the solution to (32) exists. From (32), we also conclude that

$$
\frac{\alpha(u(s))}{u(s)} u^{\prime}(s)=|y|, \quad \forall s \in\left[0, s^{*}\right],
$$

Integrating this expression over $s$, and making the (monotone) change of integration variables $\nu=u(s)$, we obtain

$$
\int_{V(x)}^{u(\sigma)} \frac{\alpha(\nu)}{\nu} d \nu=\sigma|y|, \quad \forall \sigma \in\left[0, s^{*}\right) .
$$

In case $s^{*}<1$, then $\lim _{\sigma \rightarrow s^{*}} u(\sigma)=+\infty>V(x+s y)$ and we conclude that

$$
\int_{V(x)}^{V(x+s y)} \frac{\alpha(\nu)}{\nu} d \nu \leq \int_{V(x)}^{\infty} \frac{\alpha(\nu)}{\nu} d \nu=s^{*}|y| \leq|y| .
$$

Otherwise, using (33) and (34) with $\sigma=1$, we also conclude than

$$
\begin{aligned}
\int_{V(x)}^{V(x+s y)} \frac{\alpha(\nu)}{\nu} d \nu=\int_{V(x)}^{v(1)} \frac{\alpha(\nu)}{\nu} d \nu & \\
& \leq \int_{V(x)}^{u(1)} \frac{\alpha(\nu)}{\nu} d \nu \leq|y| .
\end{aligned}
$$

Since $V(x+y) \geq V(x)$ and $\alpha$ is monotone, we have that

$$
\alpha(\nu) \geq \alpha(V(x)), \quad \forall \nu \in[V(x), V(x+y)] .
$$

Moreover, since $|x| \geq \bar{\chi}(|y|)$, we conclude from this and (31) that

$$
\alpha(\nu) \geq \alpha(V(x)) \geq-L|y| / d, \quad \forall \nu \in[V(x), V(x+y)] .
$$

and therefore

$$
\begin{aligned}
|y| \geq \int_{V(x)}^{V(x+y)} \frac{\alpha(\nu)}{\nu} d \nu \geq-\frac{|y|}{d} \int_{V(x)}^{V(x+y)} \frac{1}{\nu} d \nu \\
=-\frac{|y|}{d} \log \frac{V(x+y)}{V(x)},
\end{aligned}
$$

from which one concludes that

$$
V(x+y) \leq e^{-d} V(x) .
$$

Since $d<0$, this is also true when $V(x+y)<V(x)$. So far we have shown that, for every $d<0$, there exists a function $\bar{\chi} \in \mathcal{K}_{\infty}$ such that

$$
|x| \geq \bar{\chi}(|y|) \quad \Rightarrow \quad V(x+y) \leq e^{-d} V(x), \quad \forall x, y \in \mathbb{R}^{n} .
$$

Suppose now that $|x|<\bar{\chi}(|y|)$. Since $V$ is continuous and zero at zero, there exists a function $\alpha_{2} \in \mathcal{K}_{\infty}$ such that

$$
\begin{aligned}
V(x+y) \leq \alpha_{2}(|x+y|) & \leq \alpha_{2}(|x|+|y|) \\
& \leq \alpha_{2}(\bar{\chi}(|y|)+|y|)=: \chi(|y|),
\end{aligned}
$$

with $\chi \in \mathcal{K}_{\infty}$. Therefore, no matter what is the value of $x$, we always have $V(x+y) \leq e^{-d} V(x)+\chi(|y|)$, from which C1 follows.

C3. We show that this condition implies C2. Defining

$$
\alpha(s):=\operatorname{essipup}_{V(x) \geq s, \nabla V(x) \neq 0} \frac{V(x)}{|\nabla V(x)|}
$$

we conclude that $\alpha$ is monotone nondecreasing and that (29) holds. Moreover, since $V(x)$ is positive definite and $\frac{V(x)}{|\nabla V(x)|}$ is radially unbounded we always conclude that $\alpha$ is radially unbounded. The above construction for $\alpha$ does not guarantee that this function is continuous. However, it is also possible to choose a continuous function in $\mathcal{K}_{\infty}$ smaller than $\alpha$.

C4. This is a straightforward consequence of C3.

\section{B. Non-expansiveness for vector fields}

The following results address ISS for arbitrarily large reverse a.d.t.

Theorem 8 (non-expansive for vector fields) Assume that $f(x, w)$ has linear growth on $w$ uniformly over $x$. A locally Lipschitz, positive definite, radially unbounded function $V$ : $\mathbb{R}^{n} \rightarrow \mathbb{R}$ is non-expansive for the vector field $f(x, w)$ if any of the following conditions holds:

C5 $\forall c<0$, there exists a function $\bar{\chi} \in \mathcal{K}_{\infty}$ such that

$$
|\nabla V(x)| \cdot|y| \leq-c V(x)+\bar{\chi}(|y|), \quad \forall x, y \text { a.e. }
$$

C6 there exists a function $\alpha \in \mathcal{K}_{\infty}$ such that ${ }^{9}$

$$
\alpha(V(x))|\nabla V(x)| \leq V(x), \quad \forall x \text { a.e. }
$$

C7 $\frac{V(x)}{|\nabla V(x)|}$ is radially unbounded a.e.

$$
\begin{aligned}
& { }^{9} \mathrm{Si} \\
& \text { (36). }
\end{aligned}
$$




$$
V(x)=\sum_{i=1}^{m}\left(x^{T} P_{i} x\right)^{i}, \quad P_{i} \geq 0, i \in\{1, \ldots, m\}
$$

where $m$ is a positive integer.

Corollary 9 (GES vs. slow-switching ISS) Impulsive systems of the following form are always slow-switching ISS:

$$
\begin{cases}\dot{x}=f(x, w), & t \neq t_{k}, k=1,2, \ldots \\ x=g_{1}\left(x^{-}\right)+g_{2}(w), & t=t_{k}, k=1,2, \ldots,\end{cases}
$$

where $x(k+1)=g_{1}(x(k))$ is globally exponentially stable, $g_{1}$ is globally Lipschitz, $g_{2}(0)=0$, and $f(x, w)$ has linear growth on $w$ uniformly over $x$.

ProOF OF COROLlary 9 Under the assumptions of the theorem, it is not difficult to verify that, for some integer $k>0$ sufficiently large there exists $a_{1}>0$ such that the function $V(x):=\sum_{i=0}^{k}|\phi(i, x)|$ is globally Lipschitz and satisfies

$$
|x| \leq V(x) \leq a_{1}|x|
$$

and

$$
V\left(g_{1}(x)\right)-V(x) \leq-\frac{1}{2}|x|,
$$

where $\phi\left(k, x_{0}\right)$ denotes the solution to $x(k+1)=g_{1}(x(k))$ at time $k$ starting at $x(0)=x_{0}$. Since $V$ is globally Lipschitz, there exists a constant $a_{2}>0$, such that $|\nabla V(x)| \leq a_{2}$ a.e. We thus conclude from condition $\mathrm{C} 7$ that $V$ is non-expansive for the vector field $f(x, w)$. On the other hand, we saw in Theorem 6 that condition C7 implies that (28) holds with arbitrarily small $d>0$. This means that for any $\bar{d}>0$, we can find an appropriate $\bar{\chi} \in \mathcal{K}_{\infty}$, so that

$$
\begin{array}{r}
V\left(g_{1}(x)+g_{2}(w)\right) \leq e^{-\bar{d}} V\left(g_{1}(x)\right)+\bar{\chi}(|w|) \\
\leq e^{-\bar{d}}\left(V(x)-\frac{1}{2}|x|\right)+\bar{\chi}(|w|) \\
\leq e^{-\bar{d}}\left(1-\frac{1}{2 a_{1}}\right) V(x)+\bar{\chi}(|w|),
\end{array}
$$

from which (6) follows provided that we choose $\bar{d}$ sufficiently small so that $e^{-\bar{d}}\left(1-\frac{1}{2 a_{1}}\right)<1$.

Proof of Theorem 8. C5. Consider an arbitrary $c<0$. Using the fact that $f(x, w)$ has linear growth and (35), we can write

$$
\begin{array}{r}
\nabla V(x) \cdot f(x, w)+c V(x) \leq L|\nabla V(x)| \cdot|w|+c V(x) \\
\leq \bar{\chi}(L|w|)=: \chi(|w|),
\end{array}
$$

from which (27) follows.

C6. We show that this condition implies C5. To this effect, for an arbitrary $c<0$, let $\chi \in \mathcal{K}_{\infty}$ be such that

$$
|x| \geq \chi(|y|) \quad \Rightarrow \quad \alpha(V(x)) \geq-|y| / c .
$$

Take some $x, y \in \mathbb{R}^{n}$ for which $|x| \leq \chi(|y|)$. Since $V$ is locally Lipschitz there exists a function $\alpha_{2} \in \mathcal{K}_{\infty}$ and a constant $k \geq 0$ such that

$$
|\nabla V(x)| \leq k+\alpha_{2}(|x|), \quad \forall x \text { a.e. }
$$

[5]. Therefore

$$
\begin{aligned}
|\nabla V(x)| \cdot|y| & \leq k|y|+\alpha_{2}(|x|)|y| \\
& \leq k|y|+\alpha_{2}(\chi(|y|))|y|=: \bar{\chi}(|y|),
\end{aligned}
$$

with $\bar{\chi} \in \mathcal{K}_{\infty}$ and we conclude that the inequality in (35) holds a.e. for these values of $x$ and $y$. Suppose now that $|x|>\chi(|y|)$. From (39) and (36), we conclude that

$|\nabla V(x)| \cdot|y| \leq-c \alpha(V(x))|\nabla V(x)| \leq-c V(x), \quad \forall x$ a.e.,

which shows that (35) also holds a.e. for the remaining values of $x$ and $y$.

C7, C8. Like in the proof of Theorem 6, one can show that both $\mathrm{C} 7$ and $\mathrm{C} 8$ imply C6.

\section{SUFFICIENT CONDITIONS FOR INTEGRAL ISS}

We now provide iISS counterparts to Theorems 1 and 3 . The first result establishes iISS for suitably constrained impulse time sequences under the hypotheses of Theorem 1, and the second one establishes iISS for arbitrary impulse time sequences under hypotheses weaker than (15)-(16).

Theorem 10 (uniformly iISS) Let all hypotheses of Theorem 1 hold and define the class of impulse time sequences $\mathcal{S}_{\mu, \lambda}, \mu, \lambda>0$ also as in Theorem 1. Then the system (1) is uniformly iISS over $\mathcal{S}_{\mu, \lambda}$.

Proof OF Theorem 10. From (5) and (6) we see that $V(x(t))$ is upper bounded by the (nonnegative) solution $v(t)$ of the impulsive system

$$
\begin{cases}\dot{v}=-c v+\chi(|w|), & t \neq t_{k}, k=1,2, \ldots \\ v=e^{-d} v^{-}+\chi(|w|), & t=t_{k}, k=1,2, \ldots\end{cases}
$$

with the initial condition $v\left(t_{0}\right)=V\left(x\left(t_{0}\right)\right)$. Let $z(t)$ be the (nonnegative) solution to

$$
\begin{cases}\dot{z}=\chi(|w|), & t \neq t_{k}, k=1,2, \ldots \\ z=z^{-}+\chi(|w|), & t=t_{k}, k=1,2, \ldots\end{cases}
$$

with the initial condition $z\left(t_{0}\right)=0$. Define $y:=v-z$. Then $y$ satisfies $y\left(t_{0}\right)=V\left(x\left(t_{0}\right)\right)$ and

$$
\begin{cases}\dot{y}=-c v=-c y-c z, & t \neq t_{k} \\ y=e^{-d} v^{-}-z^{-}=e^{-d} y^{-}-\left(1-e^{-d}\right) z^{-}, & t=t_{k} .\end{cases}
$$

Applying the proof of Theorem 1, with $y$ and $z$ playing the roles of $V$ and $\chi(|w|)$, respectively, we see that this impulsive system is ISS with respect to $z$ with linear gain:

$$
y(t) \leq \beta\left(y\left(t_{0}\right), t-t_{0}\right)+\gamma z(t)
$$

for some function $\beta \in \mathcal{K} \mathcal{L}$ and constant $\gamma>0$. Collecting the above formulas, we obtain

$$
\begin{gathered}
V(x(t)) \leq v(t)=y(t)+z(t) \leq \beta\left(y\left(t_{0}\right), t-t_{0}\right)+(\gamma+1) z \\
=\beta\left(V\left(x\left(t_{0}\right)\right), t-t_{0}\right)+\int_{t_{0}}^{t}(\gamma+1) \chi(|w(s)|) d s \\
+\sum_{t_{k} \in\left[t_{0}, t\right]}(\gamma+1) \chi\left(\left|w\left(t_{k}\right)\right|\right)
\end{gathered}
$$


from which the iISS estimate (4) follows.

The following result is a relatively straightforward extension of the proof of the corresponding result for continuous systems given in [2].

Theorem 11 Suppose that there exists a positive definite, radially unbounded, locally Lipschitz function $V: \mathbb{R}^{n} \rightarrow \mathbb{R}$, a positive definite function $\alpha$, and a class $\mathcal{K}_{\infty}$ function $\chi$ satisfying

$$
\nabla V(x) \cdot f(x, w) \leq-\alpha(V(x))+\chi(|w|), \quad \forall x, w \text { a.e. }
$$

and

$$
V(g(x, w)) \leq V(x)+\chi(|w|), \quad \forall x, w .
$$

Then the system (1) is uniformly iISS over all impulse time sequences $\left\{t_{k}\right\}$.

Proof of Theorem 11. From (40) and (41) we see that $V(x(t))$ is upper bounded by the (nonnegative) solution $v(t)$ of the impulsive system

$$
\begin{cases}\dot{v}=-\alpha(v)+\chi(|w|), & t \neq t_{k}, k=1,2, \ldots \\ v(t)=v^{-}(t)+\chi(|w(t)|), & t=t_{k}, k=1,2, \ldots\end{cases}
$$

with the initial condition $v\left(t_{0}\right)=V\left(x\left(t_{0}\right)\right)$. Let $z(t)$ be the (nonnegative and nondecreasing) solution of

$$
\begin{cases}\dot{z}=\chi(|w|), & t \neq t_{k}, k=1,2, \ldots \\ z(t)=z^{-}(t)+\chi(|w(t)|), & t=t_{k}, k=1,2, \ldots\end{cases}
$$

with the initial condition $z\left(t_{0}\right)=0$. Define $y(t):=v(t)-$ $z(t)$. Then $y$ is continuous everywhere, non-increasing, and

$$
\dot{y}=-\alpha(v)=-\alpha(y+z), \quad y\left(t_{0}\right)=V\left(x\left(t_{0}\right)\right) .
$$

Let

$$
\check{t}:=\min \left\{t \geq t_{0}: y(t) \leq z(t)\right\} \leq \infty
$$

(this is well defined because $z$ is right-continuous). Since $y$ is non-increasing and $z$ is nondecreasing, we have

$$
y(t) \leq z(t), \quad \forall t \geq \check{t}
$$

and

$$
y(t)>z(t), \quad \forall t \in\left[t_{0}, \check{t}\right)
$$

the latter of which implies that

$$
0 \leq y(t) \leq y(t)+z(t) \leq 2 y(t), \quad \forall t \in\left[t_{0}, \check{t}\right) .
$$

By [2, Lemma IV.2], there exist functions $\rho_{1} \in \mathcal{K}_{\infty}$ and $\rho_{2} \in \mathcal{L}$ such that $\alpha(r) \geq \rho_{1}(r) \rho_{1}(r)$ for all $r \geq 0$. Using (42) and (44), we have

$$
\dot{y} \leq-\rho_{1}(y+z) \rho_{2}(y+z) \leq-\rho_{1}(y) \rho_{2}(2 y), \quad \forall t \in\left[t_{0}, \check{t}\right) .
$$

Thus there exists a $\beta \in \mathcal{K} \mathcal{L}$ (which does not depend on our choice of trajectory) such that $y(t) \leq \beta\left(y\left(t_{0}\right), t-t_{0}\right)$ for $t \in\left[t_{0}, \check{t}\right)$. Combining this with (43), we have

$$
y(t) \leq \beta\left(y\left(t_{0}\right), t-t_{0}\right)+z(t), \quad \forall t \geq t_{0}
$$

and this yields (recalling the definition of $z$ )

$$
\begin{aligned}
& V(x(t)) \leq v(t)=y(t)+z(t) \leq \beta\left(y\left(t_{0}\right), t-t_{0}\right)+2 z(t) \\
= & \beta\left(V\left(x\left(t_{0}\right)\right), t-t_{0}\right)+\int_{t_{0}}^{t} 2 \chi(|w(s)|) d s+\sum_{t_{k} \in\left[t_{0}, t\right]} 2 \chi\left(\left|w\left(t_{k}\right)\right|\right)
\end{aligned}
$$

from which iISS follows.

\section{CONCLUSIONS}

We introduced concepts of ISS and integral-ISS to systems with impulsive effects and provided Lyapunov-based sufficient conditions to establish these properties. Converse Lyapunov results for systems with impulsive effects will be reported in a forthcoming paper. These results are based on the converse Lyapunov theorems for hybrid systems in [4].

\section{REFERENCES}

[1] D. Angeli and E. Sontag. Forward completeness, unboundedness observability, and their Lyapunov characterizations. Systems Control Lett., 38:209-217, 1999.

[2] D. Angeli, E. Sontag, and Y. Wang. A characterization of integral input-to-state stability. IEEE Trans. Automat. Control, 45:1082-1097, 2000.

[3] D. D. Bainov and P. S. Simeonov. Systems with Impulse Effect: Stability, Theory and Applications. Ellis Horwood, UK, 1989.

[4] C. Cai, A. R. Teel, and R. Goebel. Converse Lyapunov theorems and robust asymptotic stability for hyrid systems. In Proc. of the 2005 Amer. Contr. Conf., 2005.

[5] F. H. Clarke. Optimization and Nonsmooth Analysis. SIAM, Philadelphia, PA, 1990.

[6] J. P. Hespanha. Stabilization through hybrid control. In Encyclopedia of Life Support Systems. To appear.

[7] J. P. Hespanha and A. S. Morse. Stability of switched systems with average dwell-time. In Proc. 38th IEEE Conf. on Decision and Control, pages 2655-2660, 1999.

[8] J. P. Hespanha, A. Ortega, and L. Vasudevan. Towards the control of linear systems with minimum bit-rate. In Proc. of the Int. Symp. on the Mathematical Theory of Networks and Syst., Aug. 2002.

[9] Z. P. Jiang and Y. Wang. Input-to-state stability for discretetime nonlinear systems. Automatica, 37:857-869, 2001.

[10] H. K. Khalil. Nonlinear Systems. Prentice Hall, New Jersey, 3rd edition, 2002.

[11] V. Lakshmikantham and S. Leela. Differential and Integral inequalities: Theory and Applications. Volume I-Ordinary Differential Equations, volume 55 of Mathematics in Science and Engineering. Academic Press, New York, 1969.

[12] D. Liberzon. Switching in Systems and Control. Birkhäuser, Boston, 2003.

[13] D. Liberzon and J. P. Hespanha. Stabilization of nonlinear systems with limited information feedback. IEEE Trans. on Automat. Contr., 50(6):910-915, June 2005.

[14] J. L. Mancilla-Aguilar and R. A. Garcia. On converse Lyapunov theorems for ISS and iISS switched nonlinear systems. Systems Control Lett., 42:47-53, 2001.

[15] D. Nešić and A. R. Teel. Input-output stability properties of networked control systems. IEEE Trans. Automat. Control, 49:1650-1667, 2004.

[16] L. Praly and Y. Wang. Stabilization in spite of matched unmodeled dynamics and an equivalent definition of input-tostate stability. Math. Control Signals Systems, 9:1-33, 1996.

[17] E. D. Sontag. Smooth stabilization implies coprime factorization. IEEE Trans. Automat. Control, 34:435-443, 1989.

[18] E. D. Sontag. Comments on integral variants of ISS. Systems Control Lett., 34:93-100, 1998. 
[19] E. D. Sontag and Y. Wang. On characterizations of the inputto-state stability property. Systems Control Lett., 24:351-359, 1995.

[20] Y. Xu and J. P. Hespanha. Communication logics for networked control systems. In Proc. 2004 American Control Conf., pages 572-577, June 2004. 